\title{
Enhancer of trithorax/polycomb, Corto, regulates timing of hunchback gene relocation and competence in Drosophila neuroblasts
}

Terry L. Hafer ${ }^{1,2}$, Sofiya Patra ${ }^{1}$, Daiki Tagami ${ }^{1}$ and Minoree Kohwi ${ }^{1,3^{*}}$

\begin{abstract}
Background: Neural progenitors produce diverse cells in a stereotyped birth order, but can specify each cell type for only a limited duration. In the Drosophila embryo, neuroblasts (neural progenitors) specify multiple, distinct neurons by sequentially expressing a series of temporal identity transcription factors with each division. Hunchback ( $\mathrm{Hb})$, the first of the series, specifies early-born neuronal identity. Neuroblast competence to generate early-born neurons is terminated when the $h b$ gene relocates to the neuroblast nuclear lamina, rendering it refractory to activation in descendent neurons. Mechanisms and trans-acting factors underlying this process are poorly understood. Here we identify Corto, an enhancer of Trithorax/Polycomb (ETP) protein, as a new regulator of neuroblast competence.
\end{abstract}

Methods: We used the GAL4/UAS system to drive persistent misexpression of Hb in neuroblast 7-1 (NB7-1), a model lineage for which the early competence window has been well characterized, to examine the role of Corto in neuroblast competence. We used immuno-DNA Fluorescence in situ hybridization (DNA FISH) in whole embryos to track the position of the $h b$ gene locus specifically in neuroblasts across developmental time, comparing corto mutants to control embryos. Finally, we used immunostaining in whole embryos to examine Corto's role in repression of $\mathrm{Hb}$ and a known target gene, Abdominal B (Abd-B).

Results: We found that in corto mutants, the $h b$ gene relocation to the neuroblast nuclear lamina is delayed and the early competence window is extended. The delay in gene relocation occurs after $h b$ transcription is already terminated in the neuroblast and is not due to prolonged transcriptional activity. Further, we find that Corto genetically interacts with Posterior Sex Combs (Psc), a core subunit of polycomb group complex 1 (PRC1), to terminate early competence. Loss of Corto does not result in derepression of Hb or its Hox target, Abd-B, specifically in neuroblasts.

Conclusions: These results show that in neuroblasts, Corto genetically interacts with PRC1 to regulate timing of nuclear architecture reorganization and support the model that distinct mechanisms of silencing are implemented in a step-wise fashion during development to regulate cell fate gene expression in neuronal progeny.

Keywords: Neuroblast competence, Temporal identity, Hunchback, Corto, Polycomb, Nuclear lamina, Nuclear architecture

*Correspondence: mk3632@columbia.edu

${ }^{3}$ Kavli Institute for Brain Science, Columbia University, New York, NY 10027, USA

Full list of author information is available at the end of the article

\section{Background}

In both insects and mammals, neural progenitor cells generate diverse cell types in a stereotyped birth order [1-7]. Throughout development, progenitors progressively lose competence to generate early-born neural cell types as they gain competence to generate the lateborn cell types, thereby establishing organized tissue 
growth. Thus, progenitors have a limited window of time during which they are able to specify each neural cell type [8-12]. How competence is regulated is critical to understanding both normal development and for effectively harnessing stem cells therapeutically, but mechanisms underlying competence regulation are not well understood.

Drosophila neural progenitors, called neuroblasts, are a tractable model system to investigate mechanisms of competence regulation. In the embryonic ventral nerve cord (VNC), 30 bilaterally symmetric neuroblasts undergo repeated rounds of asymmetric divisions, producing a smaller ganglion mother cell that divides once again to generate postmitotic neural progeny. With each division, neuroblasts sequentially express a series of transcription factors, called temporal identity factors, Hunchback $(\mathrm{Hb}) \rightarrow$ Kruppel $(\mathrm{Kr}) \rightarrow$ Pou domain transcription factors 1 and 2 (collectively called Pdm) $\rightarrow$ Castor (Cas), which specify the neural identity of the descendent neurons [2, 13-18]. While neuroblasts express each temporal identity factor only transiently, the neurons descended from each neuroblast division maintain active expression of the temporal identity factor, thus becoming molecularly marked by the timing of their birth.

$\mathrm{Hb}$, the first of the temporal identity factor series, is a zinc-finger transcription factor that specifies early-born neural fate in multiple neuroblast lineages, similar to its mammalian homolog Ikaros, which promotes early-born identity in both the retina and the cortex $[19,20]$. In neuroblasts, $\mathrm{Hb}$ is expressed for only the first one or two divisions and is rapidly repressed by a transient expression of its repressor, Seven-up (Svp) [21, 22]. Though neuroblasts express $\mathrm{Hb}$ only briefly, they remain competent to specify $h b$-transcribing early-born neurons for several additional divisions after $h b$ is repressed in the neuroblast $[9,11,12,23]$. Thus, the "early competence window" is a representation of neuroblast potential to generate early-born neurons that are marked by endogenous $h b$ transcription, and this potential can be experimentally measured by identifying the neural progeny generated upon misexpressing $\mathrm{Hb}$ in the neuroblast progenitor. Previously, we showed that the early competence window is terminated when the $h b$ gene physically relocates within the neuroblast to the nuclear lamina [11], a gene silencing hub $[24,25]$. This movement to the nuclear lamina occurs much after $h b$ is already transcriptionally silent, and renders the $h b$ gene refractory to activation in the descendent neurons. Thus, while transcriptional repression by Svp terminates $h b$ expression within the neuroblast, physical relocation of the $h b$ gene to the nuclear lamina establishes a heritably silent gene state that terminates early competence (Fig. 1A). Little is known regarding the mechanisms underlying this second level of $h b$ silencing.

Here we identify an ETP protein Corto as a novel competence regulator. Loss of Corto results in a delay in $h b$ gene relocation to the neuroblast nuclear lamina and prolongs competence to specify early-born neural identity. Furthermore, consistent with function as an ETP protein, we found that Corto genetically interacts with Psc, a core subunit of PRC1, to terminate early competence. While polycomb (PcG) chromatin factors are well known for their role in the maintenance of gene repression of its target genes during development [26-28], loss of Corto does not result in derepression of $h b$ in late-stage neuroblasts. Together, our results provide insight into new regulators in nuclear architecture and the timing of neural progenitor competence transitions during development.

\section{Materials and methods \\ Fly lines}

Wild-type (w1118), corto ${ }^{L 1}$, corto ${ }^{420}$ (kind gift from Dr. Frédérique Peronnet, [29]), $P s c^{h 27}$ (Bloomington, BL\#5547), en-GAL4 (chrom II, [16, 30], UAS-hb [31], $h b^{H A}$ [11], UAS-corto [kind gift from Dr. Frédérique Peronnet, [29]]. Flies were raised on a standard cornmeal and molasses medium at $25 \mathrm{C}$.

\section{Immunostaining and antibodies}

We immunostained embryos following standard protocols [32]. Briefly, we fixed embryos in a one-to-one mixture N-heptane and $4 \%$ formaldehyde diluted in PEM buffer (0.1 M Pipes, $1 \mathrm{mM} \mathrm{MgS0}{ }_{4}, 2 \mathrm{mM}$ EGTA) and rocked them in at room temperature for $22 \mathrm{~min}$. The fixative solution was subsequently removed, and embryos were devitellinized by vigorous shaking in a 1:1 mixture of methanol:heptane. Devitellinized embryos were washed with PBS and incubated overnight at $4{ }^{\circ} \mathrm{C}$ in primary antibodies diluted in PBS- $0.1 \%$ Tween 20 (PBT). After washing, embryos were incubated at room

(See figure on next page.)

Fig. 1 Early competence window is prolonged in corto mutants. A Left, NB7-1 lineage with neural progeny displayed by birth order, clockwise. The neuroblast is at the center and divided into time segments during which each temporal identity factor is expressed. Right, hunchback $(h b)$ is transcriptionally repressed by transient pulse of Svp. Several divisions later, the $h b$ gene relocates to the nuclear lamina, rendering the gene refractory to activation in the neural progeny, closing the early competence window. B NB7-1 lineage early competence assay in representative corto mutant (corto ${ }^{L 1}$ ) compared to sibling (heterozygous) control embryo. C Quantification of early competence window (Eve ${ }^{+} H A^{+}$). Genotypes shown on $x$-axis. Each datapoint represents the average number of early-born (Eve ${ }^{+} \mathrm{H}^{+}$) neurons from a single NB7-1 lineage. A minimum of three embryos were quantified per genotype. Data are represented as mean \pm SD. P values represent comparisons to wild type. D Schematic summary of corto mutant competence phenotype 


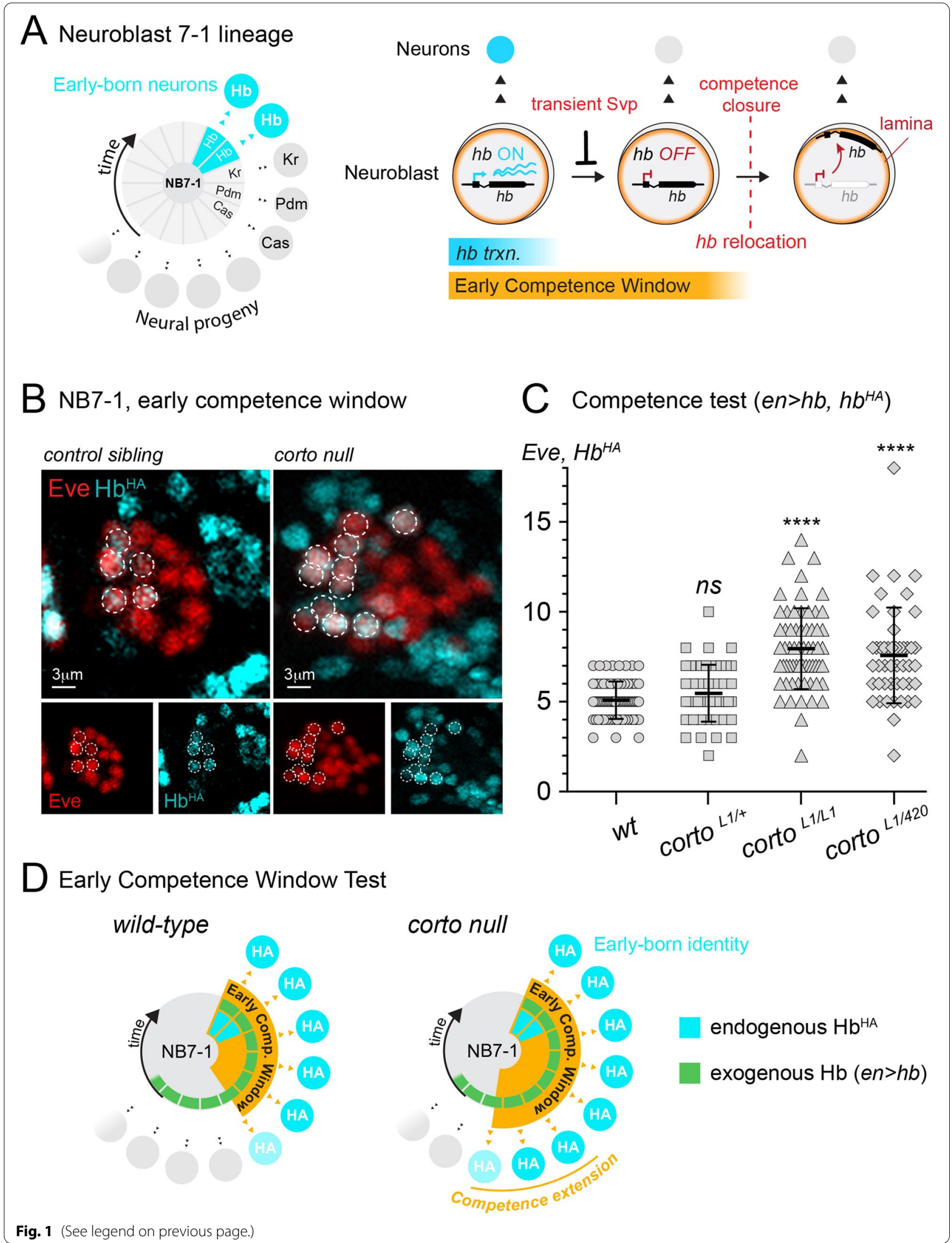


temperature in secondary antibodies for $1.5 \mathrm{~h}$, and streptavidin for $20 \mathrm{~min}$. The following primary antibodies were used: Mouse anti-Eve (clone 3C10, 1:50, Developmental Studies Hybridoma Bank (DSHB)), rat anti-HA (\#11,867,423,001, clone 3F10, 1:500, Sigma), rabbit antilamin (1:2000, kind gift from Dr. Paul Fischer); rat antiworniu (\#196,362,clone 5A3AD2, 1:200, Abcam), rat anti-dpn (\#195,172, clone 11D1CH11, 1:200, Abcam), mouse anti-PH3 (\#14,955, 1:1000, Abcam), rabbit anti$\mathrm{Hb}$ (1:200, kind gift from Dr. Chris Doe), rat anti-Zfh2 (1:200, kind gift from Dr. Chris Doe), mouse anti-AbdB (clone 1A2E9, 1:30, DSHB). Secondary antibodies against mouse, rabbit, and rat were conjugated to Alexa 488, 555, and 647 (Thermo Fisher Scientific).

\section{Immuno-DNA FISH}

DNA in situ hybridization (DNA-FISH) was performed as previously described [11, 23, 33]. Briefly, embryos were fixed in freshly-prepared $4 \%$ formaldehyde diluted in PIPES buffer $(60 \mathrm{mM} \mathrm{KCl}, 15 \mathrm{mM} \mathrm{NaCl}, 0.5 \mathrm{mM}$ spermidine, $0.15 \mathrm{mM}$ spermine, $2 \mathrm{mM}$ EDTA, $0.5 \mathrm{mM}$ EGTA, $15 \mathrm{mM}$ PIPES $\mathrm{pH}$ 7.4) and rocked for $25 \mathrm{~min}$ at room temperature with an equal volume of heptane. Embryos were devitellinized by vigorous shaking in a one-to-one solution of methanol:heptane. We generated fluorescent probes by PCR-amplifying approximately $10 \mathrm{~kb}$ of the $h b$ genomic locus [11] and using the DNA FISH Tag kit (Thermo Fisher Scientific). After rehydration, embryos were treated with RNaseA $(150 \mathrm{mg} / \mathrm{ml})$ for $2 \mathrm{~h}$ at room temperature and gradually stepped into $100 \%$ prehybridization solution (pHM: 50\% formamide; 4XSSC; $100 \mathrm{mM} \mathrm{NaH} 2 \mathrm{PO} 4, \mathrm{pH} 7.0 ; 0.1 \%$ Tween 20). After one hour of pre-hybridization at $37{ }^{\circ} \mathrm{C}$, embryos were denatured $15 \mathrm{~min}$ at $80^{\circ} \mathrm{C}$ and incubated with denatured FISH probe diluted in pre-hybridization solution overnight at $37{ }^{\circ} \mathrm{C}$ in hybridization buffer (10\% Dextran sulfate, $50 \%$ deionized formamide, 2XSSC, $0.5 \mathrm{mg} / \mathrm{ml}$ Salmon Sperm DNA). Embryos were washed at $37{ }^{\circ} \mathrm{C}$ in a series of formamide/0.3\% CHAPS solutions with decreasing formamide at each wash. After the in situ hybridization protocol was completed, embryos were subsequently immunostained according to standard immunochemistry protocol (see above), mounted in Vectashield (Vectorlabs) and imaged on a confocal microscope, Zeiss LSM 700. Additional protocol details are available upon request.

\section{Confocal imaging}

We used a Zeiss 700 Axio Imager 2 laser scanning confocal for all images. DNA-FISH images were taken at a $0.4 \mu \mathrm{m}$ step size, with pinholes that were adjusted to have equal optical section thickness in all channels.

\section{Quantification and statistical analyses}

We used standard t-tests using Prism v8. Statistical significance was classified as: ${ }^{* *}<0.01,{ }^{* * * *}<0.001,{ }^{* * * *}<0.0001$. Embryos stage 14-16 were used in competence window tests. For graphs in which n's represent individual neuroblasts, a minimum of three embryos were quantified per genotype, and for graphs in which n's represent average of single embryos, at least twelve lineages were averaged per embryo. For DNA FISH, n's represent each embryo, and number of FISH signals quantified are shown on the graph. Graphs show each data point used for statistical analysis, and bars indicate mean \pm standard deviation.

\section{Results}

Loss of Corto extends the early competence window

Neuroblasts undergo two temporally-separated, distinct types of $h b$ gene repression. In the first, $h b$ is transcriptionally repressed by Svp after one-to-two divisions. In the second, several hours and divisions later, the already transcriptionally repressed $h b$ gene physically relocates to the neuroblast nuclear lamina. This relocation causes heritable gene silencing and renders the $h b$ gene refractory to activation in the descendent neurons, thereby closing the early competence window (Fig. 1A) [11, 23]. Thus, while neuroblasts typically generate one to two early-born neurons, they are capable of producing more until nuclear architecture reorganization terminates competence. We recently identified the PcG factors as critical players in the second step, in terminating neuroblast early competence. PcG proteins are chromatin factors best known for their function to regulate gene repression through the cooperative activity of two multimeric complexes, PRC1 and PRC2 [26, 28, 34-39]. We found that Psc is required for timely $h b$ gene relocation to the neuroblast nuclear lamina and closing the early competence window [23].

Another class of proteins, the ETP proteins, has been shown to interact with the Trithorax and PcG complexes and regulate target genes. Corto, one such ETP protein, has been shown to genetically interact with PcG factors in Hox gene repression [29, 40, 41] and colocalizes with the majority of Psc-bound polytene chromosome sites [42]. Thus, we hypothesized that Corto may also function in regulating neuroblast competence. To test this, we focused on the NB7-1, one of the thirty neuroblast lineages of the embryonic VNC, for which we have markers to identify the progeny as well as have detailed knowledge of the temporal progression of its lineage and competence $[9,11,12,16,23]$. Briefly, we took advantage of the GAL4/UAS system [43] and used the engrailed GAL4 (en-GAL4) driver to drive strong, continuous Hb expression in NB7-1 throughout its entire lineage $(e n>h b)$ and determined the number of early-born neurons generated, 
a quantitative measure of the length of the early competence window. To distinguish between the endogenously activated $\mathrm{Hb}$ protein in response to early-born identity specification and the $\mathrm{Hb}$ protein misexpressed from the $U A S-h b$ construct, we implemented an endogenously encoded bacterial artificial chromosome (BAC) transgene that includes the $h b$ genomic locus and all of its regulatory enhancers. This BAC has been modified to include a hemagglutinin epitope tag (HA) fused to $\mathrm{Hb}$, and our previous work has shown that this BAC insertion is sufficient to rescue a $h b$ null animal and shows the same expression pattern as the native $h b$ gene [11]. Thus, we are able to use HA as a proxy for endogenous $\mathrm{Hb}$ expression. We co-stained $\mathrm{Hb}$ and Even skipped (Eve), a marker for the $U$ motoneuron progeny of the NB7-1 lineage, and quantified the number of early-born neurons $\left(\mathrm{Eve}^{+} \mathrm{HA}^{+}\right)$produced in response to continuous $\mathrm{Hb}$ misexpression. We note that in the competence assay, the degree of neuroblast response to $\mathrm{Hb}$ misexpression is variable even among homologous neuroblast lineages within an individual embryo. Thus, the length of the early competence window associated with a particular genotype is represented as an average of all lineages quantified. Compared to $5.1 \pm 1.0 \mathrm{Eve}^{+} \mathrm{HA}^{+}$neurons in wild type NB7-1, we found $8.0 \pm 2.3$ early-born neurons in corto $^{L 1}$ mutants $(p<0.0001)$. To control for any possible background mutations, we also examined transheterozygous animals of two independent loss of function alleles, corto $^{L 1}$ and $\operatorname{corto}^{420}$ [44] and observed consistent results, with $7.6 \pm 2.7$ early-born neurons generated $(p<0.0001)$ (Fig. 1B-D), indicating a prolonged early competence window. Thus, Corto plays an essential role in terminating early competence at mid-embryogenesis.

\section{$h b$ relocation to neuroblast nuclear lamina is delayed in corto mutant neuroblasts}

We previously showed that the early competence window closes when the $h b$ gene relocates to the neuroblast nuclear lamina at mid-embryogenesis [11, 23]. Thus, given the extension in early competence in corto mutants, we next examined $h b$ gene positioning in corto mutant neuroblasts using in vivo immuno-DNA Fluorescence in situ Hybridization (FISH). Embryos were hybridized with a fluorescent DNA probe generated against approximately $10 \mathrm{~kb}$ at the $h b$ gene locus and subsequently immunostained with Lamin Dm0, a B-type lamin intermediary filament that labels the nuclear envelope, and Worniu, a neuroblast-specific transcription factor. In corto mutant neuroblasts, we found that the proportion of $h b$ gene loci at the nuclear lamina, which we defined as FISH signals that pixel-overlapped with lamin signals, was significantly reduced at mid-embryogenesis (stage $12: 56.9 \pm 10.1 \%$ at lamina in wild type versus $33.7 \pm 6.7 \%$ in corto $^{-/}, P=0.0004$; stage $13 / 14: 56.4+6.6 \%$ at lamina in wild type versus $43.3 \pm 2.8 \%$ in corto $^{-/}, P=0.0006$ ) (Fig. 2A,B). We observed a reduction in $h b$ localization to the nuclear lamina in corto mutant neuroblasts even when we included FISH signals that were near, but not touching the lamina $(\leq 0.4 \mu \mathrm{m}$ from lamin) (Fig. 2B-ii). Interestingly, the difference between wild type and corto mutants diminished over time, and by late stage 15 , there was no discernible difference in $h b$ gene-lamina association between the genotypes (stage 15: $57.3 \pm 3.8 \%$ at lamin in wild type versus $57.0 \pm 3.3 \%$ in corto $^{-/}$ mutants, $P=$ not significant) (Fig. $2 \mathrm{~B}$ ). Thus, the data show that in corto mutants $h b$ gene relocation to the neuroblast nuclear lamina is delayed. It is worth noting that we do not observe an all-or-nothing association of the $h b$ gene with the nuclear lamina, and even at stage 11 , during the early competence window, $\sim 25 \%$ of the $h b$ gene loci are localized at the lamina. In fact, reports from others show that even genomic regions outside of laminaassociated domains are located at the periphery $\sim 30 \%$ of the time [45-47], underscoring the highly dynamic and non-static nature of the genome [48]. Importantly, we observe a two-fold increase in $h b$ gene association with the neuroblast nuclear lamina at mid-embryogenesis in wild type embryos that has functional consequences on neuroblast competence, and this relocation is delayed in corto mutants.

To confirm that the delay in $h b$ gene relocation in corto mutants is not due to a more general delay in neuroblast divisions, we co-stained embryos with Deadpan (Dpn), a pan-neuroblast marker, and phospho-histone 3 (PH3), a marker for dividing cells. There was no difference in numbers of dividing neuroblasts at either stage 12 or 14 . Embryonic neuroblasts gradually divide more slowly as neurogenesis progresses, and this dynamic was unchanged in corto mutants, suggesting that neuroblast proliferation is progressing normally (Figure S1).

Normal $\mathrm{Hb}$ temporal dynamics in corto mutant neuroblasts While genes localized at the nuclear lamina are typically in a repressed or silenced state, actively transcribed genes are often localized interiorly [49]. If the delay in $h b$ gene relocation to the neuroblast nuclear lamina in Corto mutant embryos is due to prolonged $h b$ transcription, we would expect a concomitant increase in the number of early-born neurons produced. By immunostaining for Eve, we did observe on occasion ectopic early-born neurons, but the occurrence was rare $\left(2 \mathrm{Eve}^{+} \mathrm{Hb}^{+}\right.$neurons in wild type compared to an average 2.1 in corto $^{L 1 / 420}$ mutants) (Figure S2). Drosophila embryo development is highly stereotyped, allowing us to use morphological characteristics to compare stage-matched animals of different genotypes, and we found no qualitative differences 


\section{A $h b$ DNA FISH Worniu Lamin}
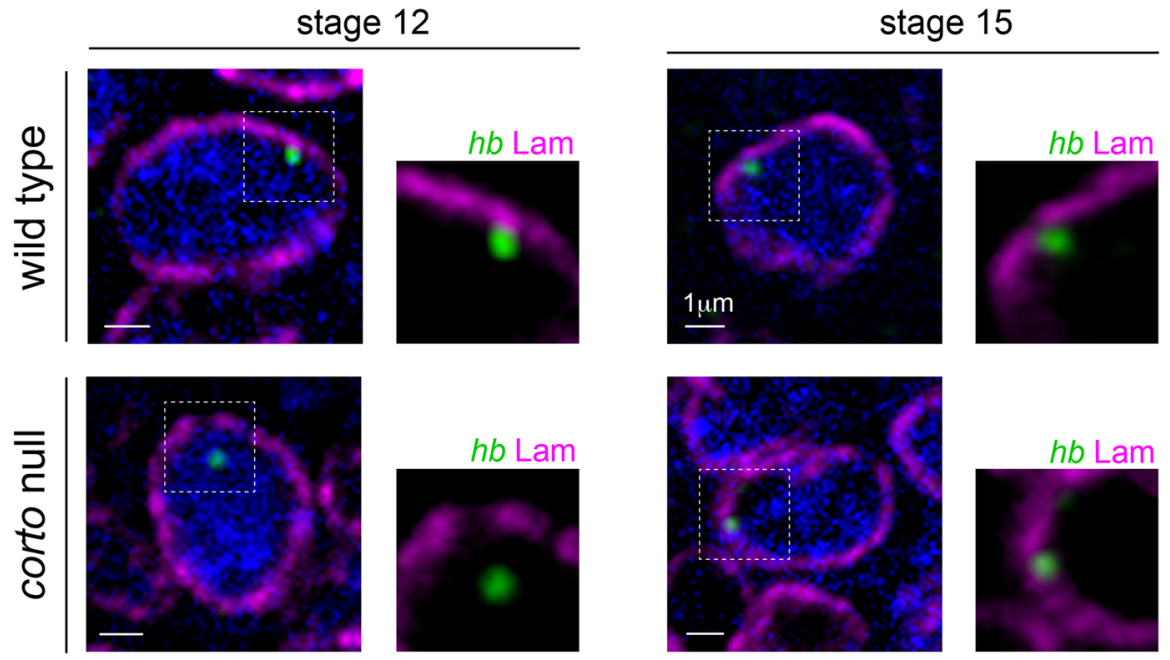

B $\%$ hb gene at neuroblast nuclear lamina
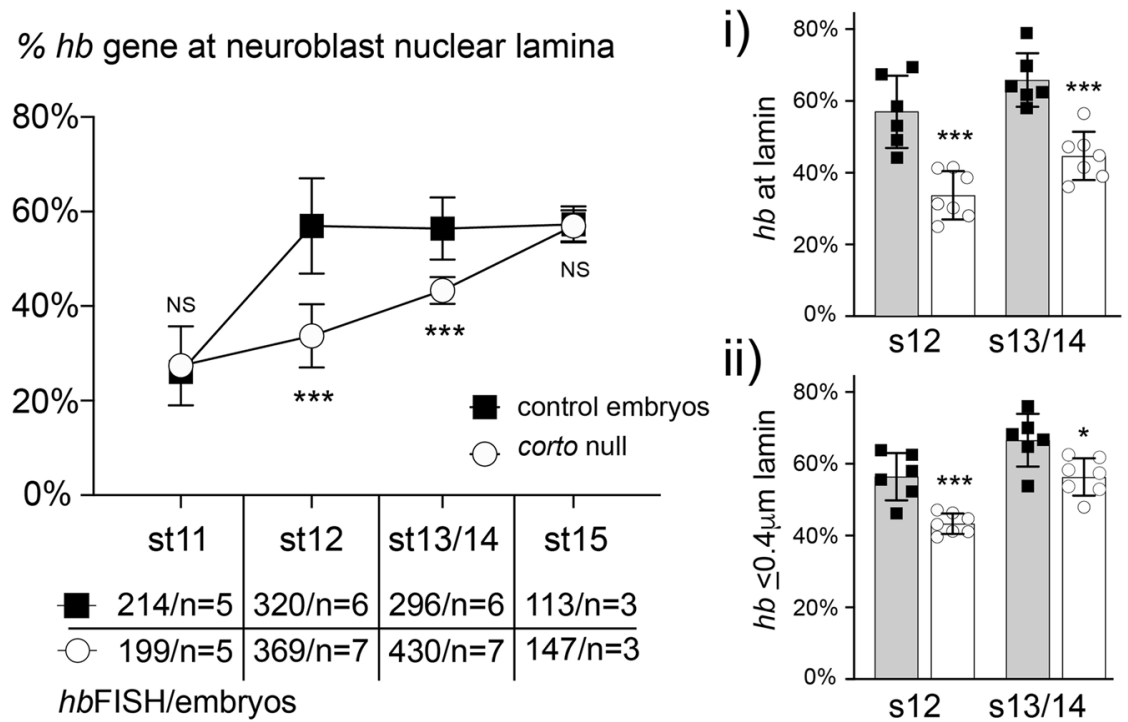

Fig. $2 \mathrm{hb}$ gene relocation to neuroblast nuclear lamina is delayed in corto mutants. A Single z-plane through nucleus of representative neuroblast from intact stage 12 and 15, wild type or corto mutant (corto ${ }^{L 1}$ ) embryos, selected at the location of the brightest part of a $h b$ DNA FISH signal (green spot). Immunostaining of lamin (magenta), and Worniu (pan-neuroblast, blue) shows position of $h b$ loci within the three-dimensional space of neuroblast nuclei. Higher magnification of $h b$ loci within neuroblast nucleus shown to the right of each FISH image; worniu is not shown for clarity. B Fraction of $h b$ loci at the nuclear lamina (pixel-overlapping with lamin signal) quantified from wild type and corto mutant embryo neuroblasts across development. Each data point in line graph is the average fraction of $h b$ loci at the lamina among multiple embryos (number of $h b$ loci and embryos quantified shown below each stage). NS, not significant. Bar graphs to the right show mean fraction of $h b$ loci either i) at lamin or ii) within $0.4 \mu \mathrm{m}$ from lamin. Each data point in bar graphs represents a single embryo. All data are represented as mean \pm SD

in the timing of $\mathrm{Hb}$ repression in neuroblasts between wild type and corto mutants (Fig. 3A). By late stage 12, $\mathrm{Hb}$ is expressed by only a few neuroblasts in the thoracic segments (Fig. 3A, arrowheads) and is not detectable in any neuroblasts at stage 13 , but rather is expressed in the neuronal layer, deeper in the embryo (Fig. 3B). Upon quantifying the proportion of $\mathrm{Hb}^{+}$neuroblasts between stage 11 and 12, when $\mathrm{Hb}$ is rapidly being repressed across the neuroblast population, we measured no difference between wild type and corto mutants, indicating $\mathrm{Hb}$ was downregulated in a timely manner (Fig. 3C). Further arguing against a strong role for Corto as a $h b$ transcriptional repressor, we found that Corto overexpression in neuroblasts had no effect on the production of earlyborn neurons (Figure S3), in contrast to $h b$ 's known transcriptional repressor, Svp, which reduces the number of 
early-born neurons upon overexpression [21]. Thus, the impaired $h b$ gene-lamina relocation phenotype in corto mutant neuroblasts we observed at stages 12-14 (7-11 h after egg lay, AEL), is well after $h b$ is already transcriptionally downregulated, and the relocation phenotype is not due to prolonged $h b$ transcription (prior to $7 \mathrm{~h} \mathrm{AEL)}$ that keeps it in the nuclear interior.

\section{Psc and Corto genetically interact to restrict the early competence window}

Corto has been reported to interact with PcG chromatin factors to maintain gene repression $[29,40]$. In particular, Corto shares many binding sites on polytene chromosomes with Psc $[40,42]$, which plays a central role in PRC1 silencing activity [27]. We asked whether Corto and Psc genetically interact to terminate competence by testing the early competence window in transheterozygous mutant embryos. We examined embryos stages 14-16, the tail end of the NB7-1 lineage, and found that while neither Psc heterozygotes or corto heterozygotes alone have a competence phenotype (P value not significant), $P s c^{h 27 /+} ;$ corto $^{L 1 /+}$ transheterozygous animals have prolonged early competence (Fig. 4A) (Psc ${ }^{h 27 /+} ;$ corto $^{L 1 /+}$ $6.4 \pm 1.5$ vs. wild type $5.4+1.1, P=0.0004)$. Given the variability in response to $\mathrm{Hb}$ misexpression even among homologous neuroblasts within the same embryo, we additionally displayed the data as a scatterplot, showing the number of early-born neurons relative to $\mathrm{Eve}^{+}$neurons, representing the total cells in the lineage upon $\mathrm{Hb}$ misexpression (Fig. 4A-ii). Psc ${ }^{h 27 /+} ;$ corto $^{L 1 /+}$ transheterozygous neuroblasts generated, overall, a higher fraction of early-born neurons than wild type neuroblasts with similar numbers of $\mathrm{Eve}^{+}$cells, indicating increased competence among neuroblasts with comparable levels of $\mathrm{Hb}$ misexpression. We note that for the stages analyzed, the number of Eve ${ }^{+}$cells is higher in older embryos, as expected (stage 14: $18.7 \pm 2.9$ Eve $^{+}$cells at stages 14, $n=68$ lineages from 5 embryos, vs stage15/16: $23.0 \pm 3.6$, $n=136$ lineages from 8 embryos, $p<0.0001$ ), whereas the number of $\mathrm{Eve}^{+} \mathrm{HA}^{+}$(early-born) neurons did not correlate with stage (stage 14: $5.9 \pm 1.5 \mathrm{Eve}^{+} \mathrm{HA}^{+}$neurons vs stage $15 / 16: 5.6 \pm 1.4$, not significant). This confirms that all embryos sampled had completed production of earlyborn neurons. Together, we conclude that Psc and Corto genetically interact to close the competence window to specify early-born neurons.
In contrast, overexpression of Corto did not have any effect on competence. While wild type animals had an average of $5.5 \pm 0.3 \mathrm{Eve}^{+} \mathrm{HA}^{+}$neurons upon $\mathrm{Hb}$ misexpression (68 hemisegments quantified from $n=4$ embryos), co-overexpression of Corto did not yield a statistically different result, $6.1 \pm 0.3 \mathrm{Eve}^{+} \mathrm{HA}^{+}$neurons $(86$ hemisegments quantified from $n=5$ embryos) (Fig. 4C). If Corto acts in neuroblasts primarily through its interactions with the PcG complex, perhaps the stoichiometry or activity level of one or more PcG complex subunits is rate-limiting.

\section{Corto does not act to maintain repression of $\mathrm{Hb}$ or the Hox gene, Abdominal $B$, in neuroblasts}

PcG factors have been well studied for their roles in maintenance of target gene repression. As an enhancer of PcG, Corto has been reported to play a similar role in the repression of Abd-B, a known PcG Hox target gene, and loss of Corto results in its derepression [41]. Our recent study [23] showed that the $h b$ intron region is a strong PcG target site, and loss of PRC1 impairs $h b$ genelamina relocation. Interestingly, however, loss of PcG does not result in $\mathrm{Hb}$ derepression in neuroblasts even in late stage embryos. Moreover, even the most severe loss of function mutants for PRC1 $\left(P s c-S u(z) 2^{P 3 C}\right)$ and PRC2 (maternal and zygotic mutants of extra sex combs, escmat/zyg $)$, did not show any neuroblast derepression of $\mathrm{Hb}$ in late-stage embryos [23], raising the possibility that PcG factors may not function in transcriptional repression in this cell type. We thus examined $\mathrm{Hb}$ expression in late-stage corto mutant embryos to determine whether $\mathrm{Hb}$ becomes derepressed. Similar to the PRC1 mutants, we also did not find $\mathrm{Hb}$ derepressed in neuroblasts of late stage corto mutant embryos (Fig. 5A). While we did observe Abd-B derepression in corto mutants, consistent with previous observations [41], surprisingly, the derepression was limited to the epithelia, and we did not find Abd-B derepressed in neuroblasts (Fig. 5B-C). In summary (Fig. 6), our results show that Corto is required for the timely relocation of the $h b$ gene to the neuroblast nuclear lamina and genetically interacts with PRC1 to terminate early competence. However, it does not play a role in maintaining $h b$ gene repression, and in a departure from its known function, it does not play a role in repression of its known target $A b d-B$, within neuroblasts.

(See figure on next page.)

Fig. 3 Temporal dynamics of $\mathrm{Hb}$ repression in neuroblasts is not affected in corto mutants. A Ventral view of VNC wild type or corto mutant $\left(\right.$ corto $\left.^{L 1}\right)$ embryos at late stage 12, when the last few neuroblasts in the thoracic segments still express $\mathrm{Hb}$ (cyan), but the rest, including all abdominal neuroblasts, are no longer $\mathrm{Hb}^{+}$. Neuroblasts are marked by pan-neuroblast marker, Dpn (red). B At stage 13, Hb is no longer detectable in any neuroblasts (top panels) but can be detected in neurons, deeper in the VNC (bottom panels). Hb shown alone in grayscale in A and B. C Quantification of fraction of neuroblasts expressing $\mathrm{Hb}$ at stage 11 and stage 12 comparing wild type and corto mutant embryos, showing similar decrease in $\mathrm{Hb}$ neuroblast expression over time. D Schematic diagram summarizing results 


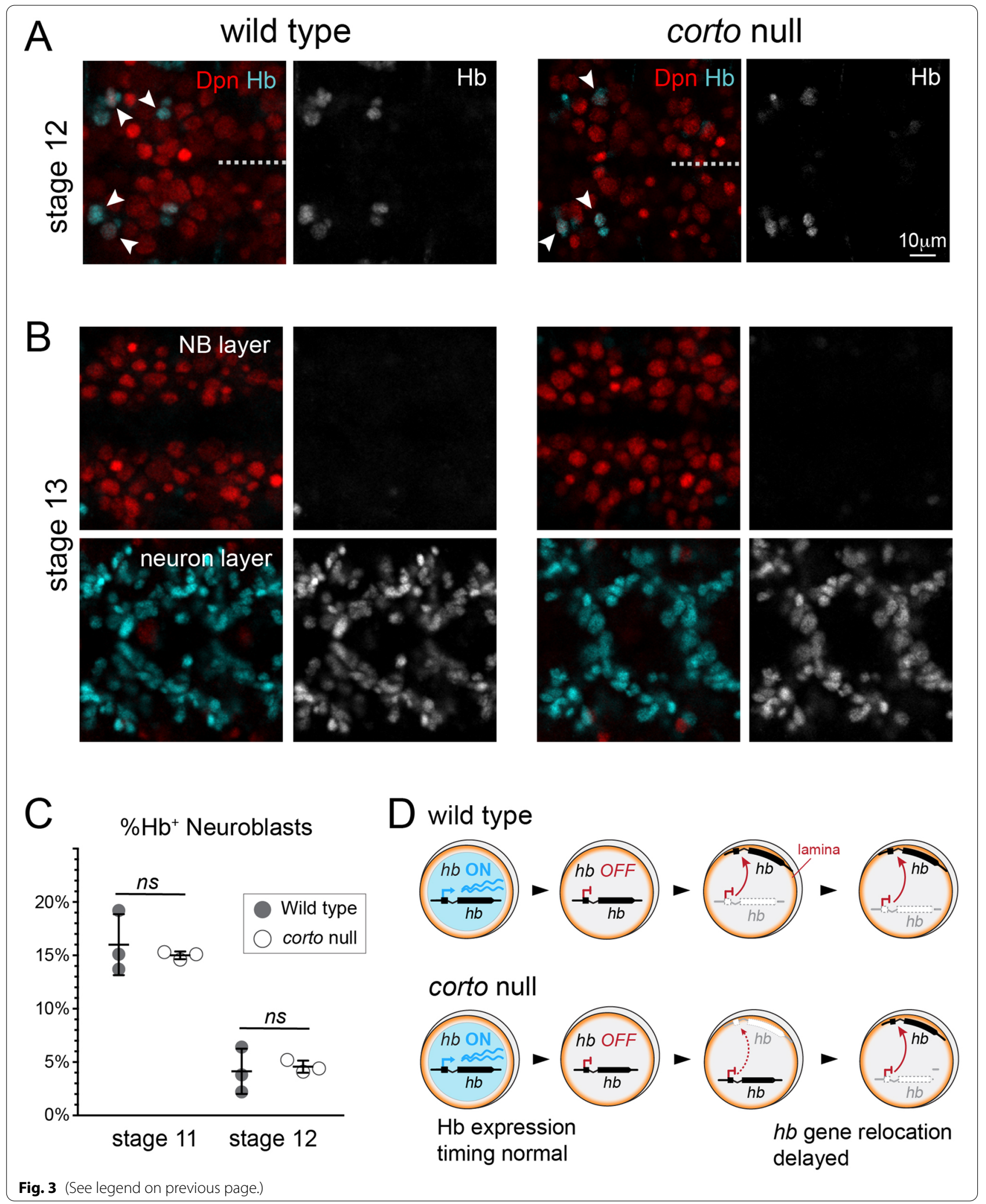



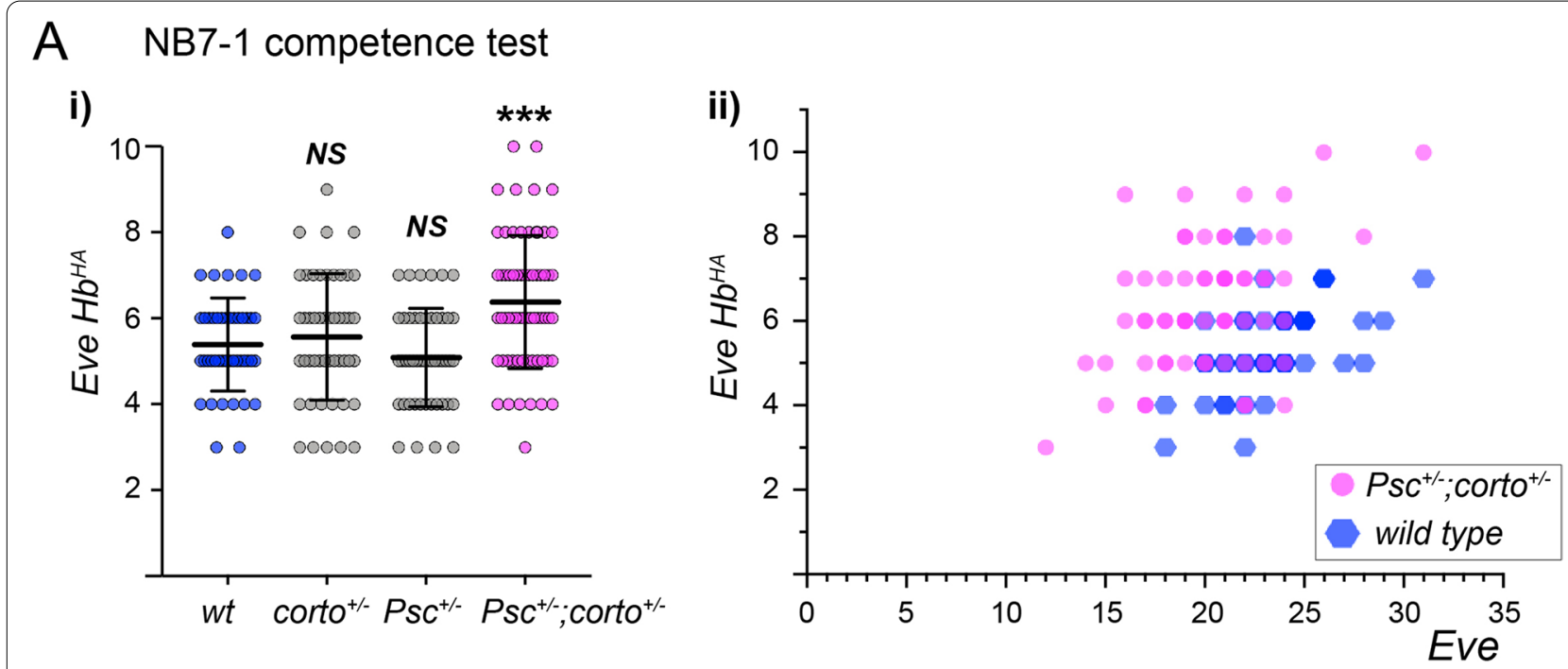

B
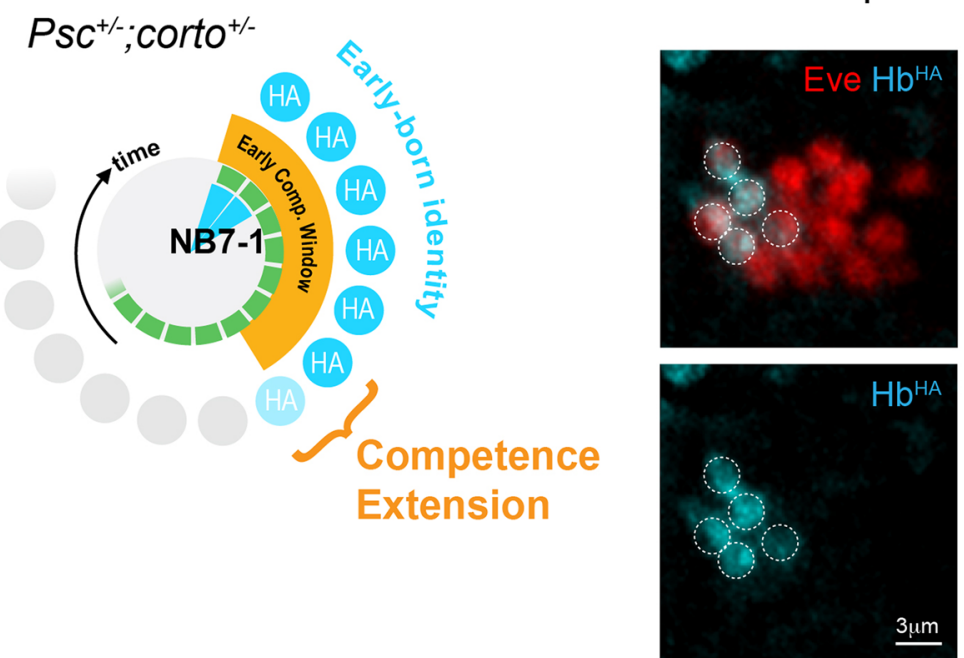

\section{Early-born neurons (Eve+Hb $\left.b^{H A+}\right)$}

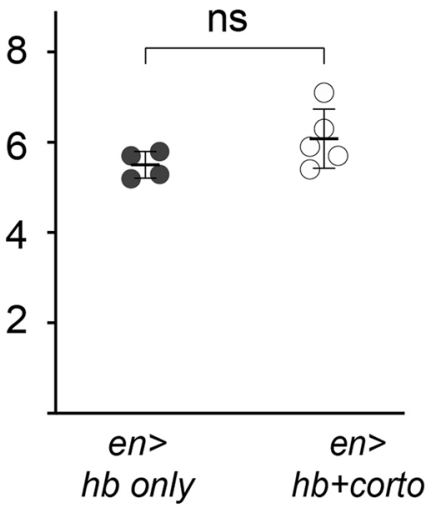

Fig. 4 Corto and Psc genetically interact to close the early competence window. A i), NB7-1 lineage early competence assay comparing wild type, corto $^{L 1 /+}, P_{S C}{ }^{h 27 /+}$, or corto ${ }^{L 1 /+} ; P_{S C} C^{27 /+}$ transheterozygous embryos. Bars represent mean \pm SD. ii), Scatterplot of wild type and corto ${ }^{L 1 /+} ; P_{S C}{ }^{27 /+}$ data, showing the number of Eve ${ }^{+} \mathrm{HA}^{+}$(early-born) neurons relative to the total number of Eve neurons within an individual NB7-1 lineage. Each data point represents competence data of a single NB7-1 lineage quantified from three to four embryos per genotype. B Schematic circular diagram depicting results. C Early competence window test with and without co-overexpression of Corto. Example NB7-1 lineage shown for Hb and Corto co-misexpressed by en-Gal4 driver, stained for $\mathrm{Hb}^{\mathrm{HA}}$ (cyan) and Eve (red). Quantification shown to the right. Each data point represents the average of at least twelve neuroblast lineages from a single embryo. Bars represent mean \pm SD

\section{Discussion}

Here we identify Corto as a regulator of neuroblast early competence in the Drosophila embryo. Loss of Corto results in a delay in $h b$ gene relocation to the nuclear lamina in neuroblasts and an extension in the early competence window. While the low numbers of extra early-born neurons found in a subset of neuroblast lineages in corto mutant embryos could indicate an occasional extension of neuroblast $\mathrm{Hb}$ expression, we did not observe any measurable changes to $\mathrm{Hb}$ transcriptional dynamics in corto mutants across the neuroblast population. In contrast, the delay in $h b$ gene relocation was robust across neuroblasts and occurred substantially after the $h b$ gene was already repressed and thus not a result of prolonged transcriptional activity. 

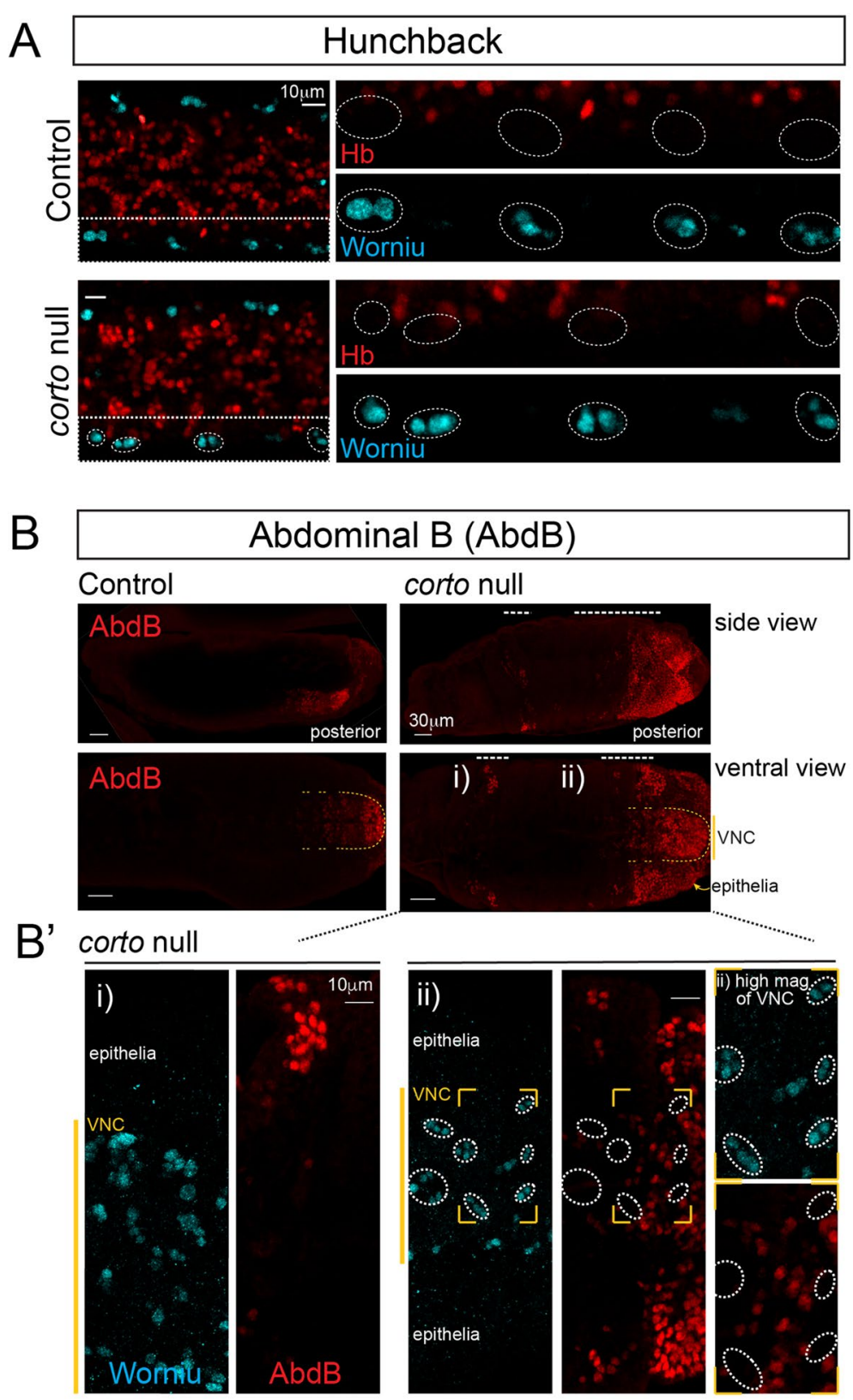

Fig. $5 \mathrm{Hb}$ and $\mathrm{Abd}-\mathrm{B}$ are not derepressed in neuroblasts in late stage corto mutant (corto ${ }^{420}$ ) embryos. A VNC of control (corto ${ }^{+/}$) or corto mutants at stage 15, stained with Worniu (pan-neuroblast marker, cyan) and $\mathrm{Hb}$ (red). Dashed box region shown as separate Worniu and $\mathrm{Hb}$ panels to the right. B Control and corto mutant whole embryos at stage 15 are shown as side and ventral views, stained with Abd-B. Dashed white lines at the top show areas of derepressed Abd-B. Yellow dashed line demarcates VNC region. B' Higher magnification of regions labeled i) and ii) in ventral view of corto mutant in B. Yellow vertical line show VNC region. Area within yellow box in ii) are shown in higher magnification to the right

In the fly embryo, neuroblasts transiently express a series of temporal identity factors which confer specific traits to the neurons descended from each division. $\mathrm{Hb}$, the first of the temporal identity factors, specifies earlyborn identity, which includes endogenous activation of the $h b$ gene within the postmitotic neuron. Misexpression of $\mathrm{Hb}$ directly in the postmitotic neuron cannot induce $h b$ transcription $[11,12]$. The molecular identity of the neuron, including its ability to activate and sustain $h b$ transcription endogenously, must be established within the neuroblast progenitor and subsequently inherited. Thus, the neuroblast "primes" the future transcriptional program that is activated by the neural progeny. Critically, the neuroblast's potential to generate an 


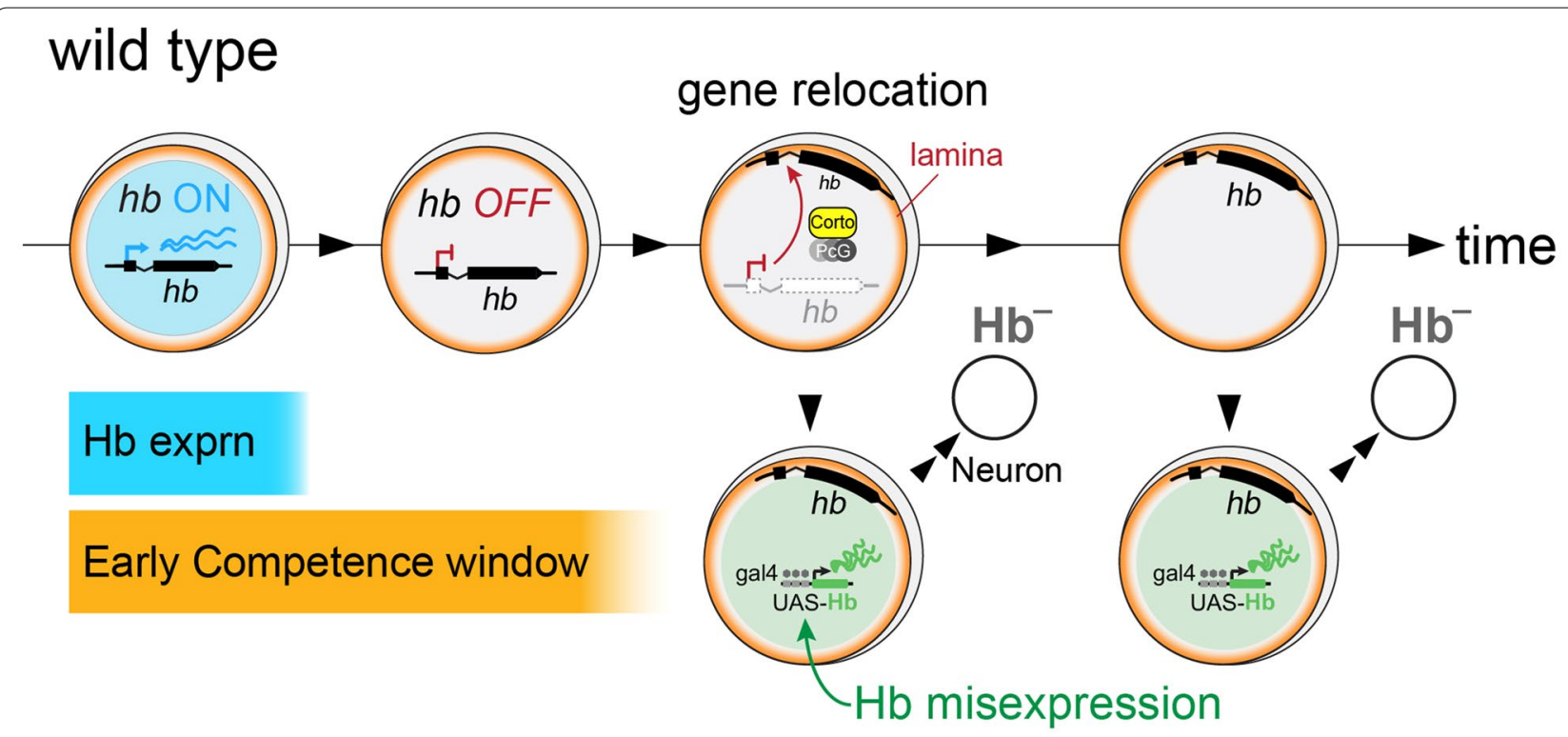

\section{corto null}

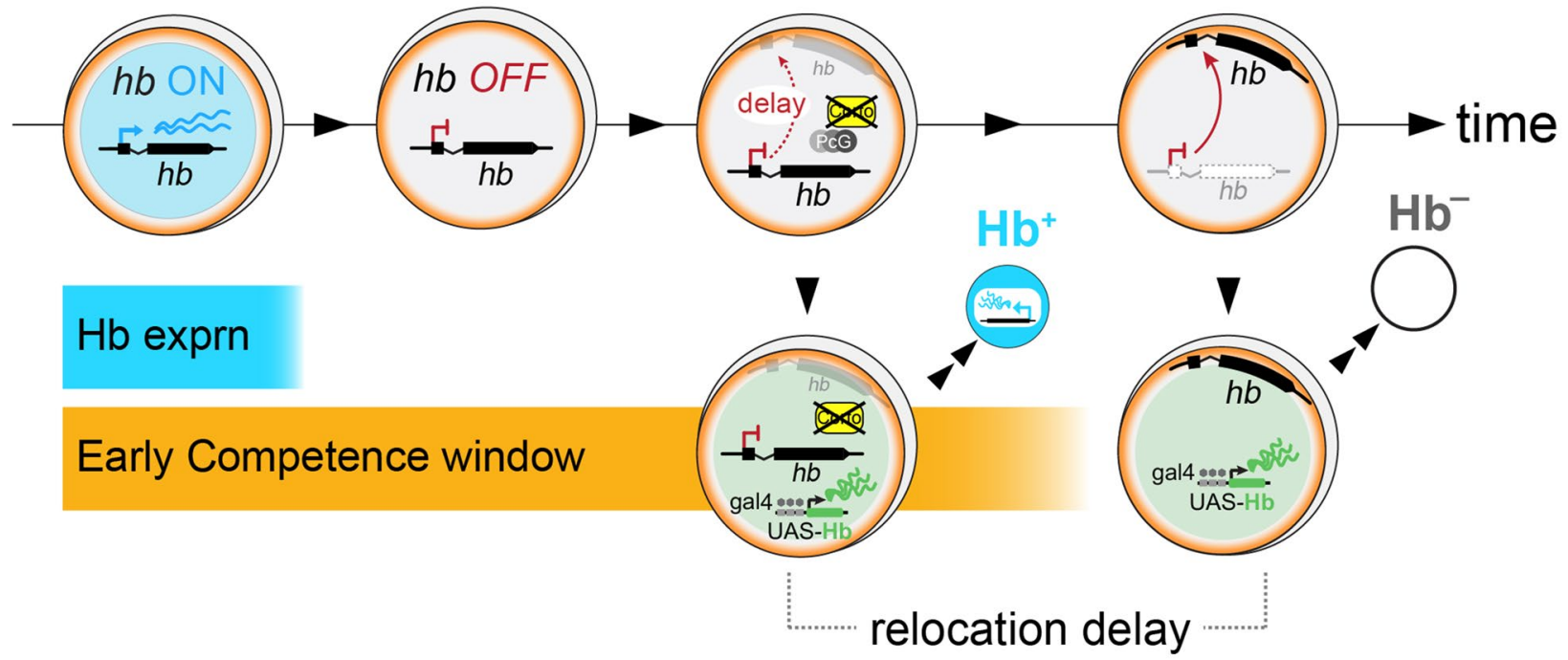

Fig. 6 Summary model. In both wild type and corto mutant neuroblasts, $h b$ transcriptional dynamics are largely normal (endogenous $h b$ depicted in blue). Much after $h b$ repression, $h b$ gene relocation to the neuroblast nuclear lamina terminates the early competence window. Loss of Corto, which genetically interacts with PcG, delays this relocation and extends early competence (misexpressed Hb depicted in green)

$h b$-transcribing early-born neuron ends not when $h b$ is transcriptionally repressed within the neuroblast, but rather several divisions later when the $h b$ gene relocates to the nuclear lamina. Upon relocation, $h b$ becomes refractory to activation within the descendent neuron, thus closing the early competence window. Corto is required for this heritable silencing through $h b$ gene relocation to the neuroblast nuclear lamina. However, relocation in corto mutants is only delayed, not completely blocked, as the $h b$ gene gradually increases association with the lamina, perhaps due to compensation by PcG factors with which it interacts. Consistent with this notion, overexpression of Corto alone did not shorten the early competence window. 
We have focused on $h b$ as a model gene to study mechanism of neuroblast competence regulation, as it is the only universally expressed gene among an otherwise diverse population of early-born neurons, but it is not clear what role, if any, $\mathrm{Hb}$ itself plays in the postmitotic, early-born neurons of the VNC. RNAi-mediated knockdown of Hb in NB7-1 neural progeny does not alter neuronal morphology or affect larval locomotor velocity [50]. We speculate that $h b$ is only one component of a broad transcriptional program in postmitotic neurons established by the nuclear architecture of the neuroblast upon specification of early-born identity, and $h b$ transcription may reflect its epigenetic state, rather than a requirement for function in these neurons. In the postembryonic brain, however, $\mathrm{Hb}$ in postmitotic neurons has been shown to play an important role in sexually dimorphic interneuron neurite morphology [51], and a recent study established a functional role for $\mathrm{Hb}$ in larval Pair1 neuron in synapse number, connectivity, and behavior [52]. It would be important for future studies to determine genelamina interactions in neuroblasts on a genome-wide scale and relate these lamina-associated genes to neuroblast competence, gene expression and neuronal function. In this context, identifying trans-acting factors of nuclear architecture and understanding the mechanisms of their function is a critical area requiring further study. Here we have found Corto, which impacts $h b$ gene relocation without affecting $h b$ transcriptional dynamics.

Though Corto has been shown to participate in PcGmediated silencing [40, 41], we did not observe any $\mathrm{Hb}$ derepression in neuroblasts in later embryonic stages, consistent with our recent observations for PcG [23]. While PcG factors bind the $h b$ gene locus and are required for $h b$ gene relocation in neuroblasts, they do not function in regulation of $h b$ transcriptional repression, a departure from their well-known roles in maintenance of target gene repression. Further supporting the lack of a repressive role in neuroblasts, overexpression of Corto did not reduce the number of early-born neurons, which is in contrast to what has been reported for Svp, $h b$ 's established transcriptional repressor [21, 22]. Additionally, PcG and Corto have been shown to be required for repression of Abd-B, the PcG Hox target gene, and similar to observation by others, we found Abd-B to be derepressed in corto mutants. Interestingly, however, while we found Abd-B derepression in the epithelia, we did not observe this in neuroblasts. Perhaps this suggests that PcG/Corto have a unique role in neuroblasts by facilitating changes to their nuclear architecture, a function independent of their better known roles in transcriptional repression. Further studies are necessary to investigate a more general role of PcG/Corto in neuroblasts comparing genome architecture and transcriptional regulation, and understand how such activity underlie neuroblast competence to determine neuronal identity and function.

\section{Conclusions}

Increasing evidence points to an important role for three dimensional organization of genome architecture in regulating transcriptional and competence states of progenitors during animal development $[53,54]$. We have identified Corto, an ETP class chromatin factor, as a new regulator of neuroblast competence. Corto is required for the timely relocation of the $h b$ gene to the neuroblast nuclear lamina and genetically interacts with Psc to close the early competence window. In neuroblasts, loss of Corto does not impact $h b$ transcriptional dynamics nor does it cause derepression of Abd-B, a departure from its known role in Abd-B repression. By identifying new chromatin regulators of competence, our results provide further mechanistic insights into how progenitor competence is regulated in vivo. Together, the results show that multiple, distinct repressive mechanisms sequentially operate in a step-wise fashion as neuroblasts age over time to terminate neuroblast competence to specify early-born neural identity.

\section{Supplementary Information}

The online version contains supplementary material available at https://doi. org/10.1186/s13064-022-00159-3.

\section{Additional file 1.}

\section{Acknowledgements}

We thank Dr. Frédérique Peronnet (French National Centre for Scientific Research) for the Corto fly stocks. We thank Dr. Paul Fisher (Stonybrook University) for the lamin antibody. We thank Dr. Gary Struhl (Columbia University) for helpful discussion on the science. We thank Dr. Natalia Molotkova for technical assistance. We thank Drs. Tanguy Lucas and Rui Galvão for critical reading of the manuscript. We thank the DSHB for antibodies and the Bloomington stock center (NIH P400D018537) for fly stocks.

\section{Authors' contributions}

T.L.H. and M.K. designed the research. T.L.H., S.P., D.T., and MK executed the experiments, collected and analyzed the data. T.L.H. and M.K. wrote the manuscript. All authors read and approved the final manuscript.

\section{Funding}

This work was supported by the NICHD (R00HD072035 and HD092381), the Rita Allen Foundation, and the Whitehall Foundation.

\section{Availability of data and materials}

No datasets or new fly stocks were generated. Fly stocks used in this manuscript are available from public stock centers or will be provided upon request to corresponding author.

\section{Declarations}

Ethics approval and consent to participate Not applicable, as no vertebrate or human subjects were used in this manuscript. 


\section{Consent for publication}

All authors approved this manuscript

\section{Competing interests}

The authors declare no competing interests.

\section{Author details}

'Department of Neuroscience, Mortimer B. Zuckerman Institute Mind Brain Behavior, Columbia University, New York, NY 10027, USA. ${ }^{2}$ Present Address: Molecular and Cellular Biology Program, University of Washington, Seattle, WA 98195, USA. ${ }^{3}$ Kavli Institute for Brain Science, Columbia University, New York, NY 10027, USA.

\section{Received: 24 December 2021 Accepted: 27 January 2022}

Published online: 17 February 2022

\section{References}

1. Brody T, Odenwald WF. Cellular diversity in the developing nervous system: a temporal view from Drosophila. Development. 2002;129(16):3763-70.

2. Kohwi M, Doe CQ. Temporal fate specification and neural progenitor competence during development. Nat Rev Neurosci. 2013;14(12):823-38.

3. Livesey FJ, Cepko CL. Vertebrate neural cell-fate determination: lessons from the retina. Nat Rev Neurosci. 2001;2(2):109-18.

4. McConnell SK. Fates of visual cortical neurons in the ferret after isochronic and heterochronic transplantation. J Neurosci. 1988:8(3):945-74.

5. Okano H, Temple S. Cell types to order: temporal specification of CNS stem cells. Curr Opin Neurobiol. 2009:19(2):112-9.

6. Rossi AM, Fernandes VM, Desplan C. Timing temporal transitions during brain development. Curr Opin Neurobiol. 2017:42:84-92.

7. Thor S. Nervous System Development: Temporal Patterning of Large Neural Lineages. Curr Biol. 2017;27(10):R392-4.

8. Cepko CL, et al. Cell fate determination in the vertebrate retina. Proc Natl Acad Sci U S A. 1996:93(2):589-95.

9. Cleary MD, Doe CQ. Regulation of neuroblast competence: multiple temporal identity factors specify distinct neuronal fates within a single early competence window. Genes Dev. 2006;20(4):429-34.

10. Hirabayashi Y, et al. Polycomb limits the neurogenic competence of neural precursor cells to promote astrogenic fate transition. Neuron. 2009;63(5):600-13.

11. Kohwi M, et al. Developmentally regulated subnuclear genome reorganization restricts neural progenitor competence in Drosophila. Cell. 2013;152(1-2):97-108.

12. Pearson BJ, Doe CQ. Regulation of neuroblast competence in Drosophila. Nature. 2003:425(6958):624-8.

13. Baumgardt $M$, et al. Neuronal subtype specification within a lineage by opposing temporal feed-forward loops. Cell. 2009;139(5):969-82.

14. Brody T, Odenwald WF. Programmed transformations in neuroblast gene expression during Drosophila CNS lineage development. Dev Biol. 2000:226(1):34-44

15. Grosskortenhaus R, et al. Regulation of temporal identity transitions in Drosophila neuroblasts. Dev Cell. 2005:8(2):193-202.

16. Isshiki T, et al. Drosophila neuroblasts sequentially express transcription factors which specify the temporal identity of their neuronal progeny. Cell. 2001;106(4):511-21.

17. Grosskortenhaus R, Robinson KJ, Doe CQ. Pdm and Castor specify late-born motor neuron identity in the NB7-1 lineage. Genes Dev. 2006:20(18):2618-27.

18 Bahrampour S, et al. Neural Lineage Progression Controlled by a Temporal Proliferation Program. Dev Cell. 2017;43(3):332-348e4.

19. Alsio JM, et al. Ikaros promotes early-born neuronal fates in the cerebral cortex. Proc Natl Acad Sci USA. 2013;110(8):E716-25.

20. Elliott J, et al. Ikaros confers early temporal competence to mouse retinal progenitor cells. Neuron. 2008;60(1):26-39.

21. Kanai Ml, Okabe M, Hiromi Y. seven-up Controls switching of transcription factors that specify temporal identities of Drosophila neuroblasts. Dev Cell. 2005;8(2):203-13.

22. Mettler U, Vogler G, Urban J. Timing of identity: spatiotemporal regulation of hunchback in neuroblast lineages of Drosophila by Seven-up and Prospero. Development. 2006;133(3):429-37.
23. Lucas T, et al. Discrete cis-acting element regulates developmentally timed gene-lamina relocation and neural progenitor competence in vivo. Dev Cell. 2021:56(18):2649-2663.e6.

24. Shevelyov YY, Nurminsky DI. The Nuclear Lamina as a Gene-silencing Hub. Curr Issues Mol Biol. 2011;14(1):27-38.

25. van Steensel B, Belmont AS. Lamina-associated domains: links with chromosome architecture, heterochromatin, and gene repression. Cell. 2017;169(5):780-91.

26. Grossniklaus U, Paro R. Transcriptional silencing by polycomb-group proteins. Cold Spring Harb Perspect Biol. 2014;6(11):a019331.

27. King IF, et al. Analysis of a polycomb group protein defines regions that link repressive activity on nucleosomal templates to in vivo function. Mol Cell Biol. 2005;25(15):6578-91.

28. Schwartz YB, et al. Genome-wide analysis of polycomb targets in Drosophila melanogaster. Nat Genet. 2006;38(6):700-5.

29. Lopez A, et al. corto genetically interacts with Pc-G and trx- $\mathrm{G}$ genes and maintains the anterior boundary of ultrabithorax expression in Drosophila larvae. Mol Genet Genomics. 2001:266(4):572-83.

30. Harrison DA, et al. Activation of a Drosophila Janus kinase (JAK) causes hematopoietic neoplasia and developmental defects. EMBO J. 1995;14(12):2857-65.

31. Wimmer EA, et al. Bicoid-independent formation of thoracic segments in Drosophila. Science. 2000;287(5462):2476-9.

32. Rothwell W.F.a.S., W., Fluorescent analysis of Drosophila embryos Drosophila Protocols. 2000, Cold Spring Harbor: Cold Spring Harbor Press.

33. Bantignies $F$, et al. Polycomb-dependent regulatory contacts between distant Hox loci in Drosophila. Cell. 2011:144(2):214-26.

34 Negre N, et al. Chromosomal distribution of PcG proteins during Drosophila development. PLoS biology. 2006:4(6):e170.

35. Schuettengruber B, Cavalli G. Recruitment of polycomb group complexes and their role in the dynamic regulation of cell fate choice. Development. 2009;136(21):3531-42

36. Simon J, Chiang A, Bender W. Ten different Polycomb group genes are required for spatial control of the abdA and $\mathrm{AbdB}$ homeotic products. Development. 1992;114(2):493-505.

37. Struhl G. A gene product required for correct initiation of segmental determination in Drosophila. Nature. 1981;293(5827):36-41.

38. Tolhuis B, et al. Genome-wide profiling of PRC1 and PRC2 Polycomb chromatin binding in Drosophila melanogaster. Nat Genet. 2006;38(6):694-9.

39. Beuchle D, Struhl G, Muller J. Polycomb group proteins and heritable silencing of Drosophila Hox genes. Development. 2001;128(6):993-1004.

40. Salvaing J, et al. The Drosophila Corto protein interacts with Polycomb-group proteins and the GAGA factor. Nucleic Acids Res. 2003;31(11):2873-82

41 Salvaing J, et al. The enhancer of trithorax and polycomb corto interacts with cyclin G in Drosophila. PLoS One. 2008:3(2):e1658.

42. Kodjabachian $L$, et al. Mutations in ccf, a novel Drosophila gene encoding a chromosomal factor, affect progression through mitosis and interact with Pc-G mutations. EMBO J. 1998:17(4):1063-75.

43. Brand $\mathrm{AH}$, Perrimon N. Targeted gene expression as a means of altering cell fates and generating dominant phenotypes. Development. 1993;118(2):401-15

44. Mouchel-Vielh E, et al. Involvement of the MP1 scaffold protein in ERK signaling regulation during Drosophila wing development. Genes Cells. 2008;13(11):1099-111.

45. Guelen $\mathrm{L}$, et al. Domain organization of human chromosomes revealed by mapping of nuclear lamina interactions. Nature. 2008;453(7197):948-51.

46. Harr JC, et al. Directed targeting of chromatin to the nuclear lamina is mediated by chromatin state and A-type lamins. J Cell Biol. 2015;208(1):33-52.

47. Zullo JM, et al. DNA sequence-dependent compartmentalization and silencing of chromatin at the nuclear lamina. Cell. 2012;149(7):1474-87.

48. Kind J, et al. Genome-wide maps of nuclear lamina interactions in single human cells. Cell. 2015;163(1):134-47.

49 Meister $\mathrm{P}$, et al. The spatial dynamics of tissue-specific promoters during C. elegans development. Genes Dev. 2010;24(8):766-82.

50. Hirono $\mathrm{K}$, et al. The Hunchback temporal transcription factor establishes, but is not required to maintain, early-born neuronal identity. Neural Dev. 2017;12(1):1.

51. Goto J, et al. Sexually dimorphic shaping of interneuron dendrites involves the hunchback transcription factor. J Neurosci. 2011;31(14):5454-9. 
52. Lee KM, Linskens AM, Doe CQ. Hunchback activates Bicoid in post-mitotic Pair1 neurons to regulate synapse number. bioRxiv: the preprint server for biology. Cold Spring Harbor: Cold Spring Harbor Laboratory; 2021.

53. Alexander JM, Lomvardas S. Nuclear architecture as an epigenetic regulator of neural development and function. Neuroscience. 2014;264:39-50.

54. Lucas T, Kohwi M. From insects to mammals: regulation of genome architecture in neural development. Curr Opin Neurobiol. 2019;59:146-56.

\section{Publisher's Note}

Springer Nature remains neutral with regard to jurisdictional claims in published maps and institutional affiliations.

- fast, convenient online submission

- thorough peer review by experienced researchers in your field

- rapid publication on acceptance

- support for research data, including large and complex data types

- gold Open Access which fosters wider collaboration and increased citations

- maximum visibility for your research: over 100M website views per year

At BMC, research is always in progress.

Learn more biomedcentral.com/submissions 